\title{
Qualité des milieux aquatiques continentaux : intérêts de l'utilisation d'un marqueur biochimique chez le poisson
}

\author{
P. Flammarion et $\mathbf{J}$. Garric \\ Laboratoire d'écotoxicologie Cemagref de Lyon
}

\section{INTRODUCTION}

La multiplication des activités humaines a entrâné la dispersion massive de micropolluants divers dans l'environnement. Ce dernier a ainsi hérité de métaux, pesticides, hydrocarbures, phtalates, détergents... tous potentiellement dangereux pour les organismes vivants des écosystèmes áquatiques.

Lorsque l'organisme ne peut pas ou ne peut plus se défendre, des effets toxiques variés se développent en fonction du temps d'exposition, de la toxicité et de la concentration des polluants dans le milieu. Mais auparavant tout commence au sein de l'organisme à un niveau cellulaire ou subcellulaile entre le composé toxique et les composants fonctionnels de la cellule : enzymes, métabolites, membranes... où ces polluants vont être pris en charge par les processus naturels de défense de l'organisme: biotransformation, élimination, bioaccumulation.

Pour évaluer la présence et l'impact de polluants dans les écosystèmes, il est ainsi possible de mesurer d'une part les effets sur les populations et communautés et d'autre part les réactions de défense des individus. La mesure de ces variables biologiques vient en complément des mesures physico-chimiques traditionnelles.

\section{BIOINDICATEURS ET BIOMAR- QUEURS}

\begin{abstract}
Les indicateurs biocénotiques ou écologiques sont des mesures, de l'état de santé de l'écosystène, au niveau des populations ou des communautés d'organismes. Cette approche est justifiée par le fait que les populations se développent dans les milieux qui leur sont favorables. Lorsque les conditions écologiques sont altérées, l'organisation naturelle des communautés aquatiques est perturbée (baisse de la diversité et/ou de l'abondance).

Le terme de biomarqueur désigne quant à lui la mesure d'une perturbation atu niveau cellulaire et tissulaire cles organismes (indicateurs biochimiques, physiologiques on histologiques d'exposition d'un organisme à des xénobiotiques [1]).

En particulier; les biomarqueurs biochimiques sont des mesures de réactions biologiques impliquées dans les phases précoces de l'intoxication: l'observation de changements biochimiques permet de détecter une perturbation chez les organismes avant l'apparition de signes pathologiques irréversibles (dégâts tissulaires; nécroses, tumeurs...) et avant l'affablissement des populations (taux de croissance,
\end{abstract}

Continental aquatic environment quality: use of tish biochemical markers

Biological assessment is wefli in addition to conventional chemical nesures in order to monitor the water quality. Biochemical markens are early waming Sutens of lhe exposition of orsansm to pollutants. Among them. the induction of we fish EROD ach w enables the detection of specife compounds PAHs, $P C B$, lloxines

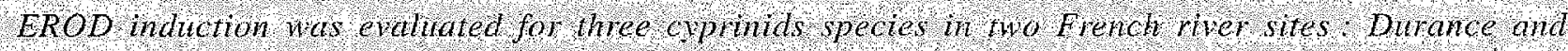
Moselte in fle river Dutance, the closer to a chemical plant the sampling site was, the higher the meast red EROD nduction. Wh the river Moselle the fish showed a signiffant indiced LROD activity after indus: trial effuent discharges. In both cases the measwe has spectes lependent.

Although the EROD activy sems a fitted tool, to assess the water ghality complementary stidies are necessany to understand the effech of biotic and ubiofic variables on the resulis. 
Tableau 1. - Avantages et inconvénients des différentes méthodes d'évaluation in situ présentées (d'après [3]).

\begin{tabular}{|l|l|l|}
\hline \multicolumn{1}{|c|}{ Avantages } & \multicolumn{1}{c|}{ Inconvénients } \\
\hline $\begin{array}{l}\text { Analyses } \\
\text { chimiques }\end{array}$ & $\begin{array}{l}\text { information spécifique, } \\
\text { quantitative, } \\
\text { sensible }\end{array}$ & $\begin{array}{l}\text { méconnaissance des effets toxiques et des } \\
\text { interactions entre composés, } \\
\text { méconnaissance de la signification biologique } \\
\text { des informations obtenues }\end{array}$ \\
\hline $\begin{array}{l}\text { Marqueurs } \\
\text { biochimiques }\end{array}$ & $\begin{array}{l}\text { spécificité, } \\
\text { signification toxicologique, } \\
\text { alarme précoce }\end{array}$ & \begin{tabular}{l} 
signification écologique imprécise \\
\hline $\begin{array}{l}\text { Indicateurs } \\
\text { écologiques } \\
\text { mise en évidence d'un stress ou } \\
\text { d'une perturbation }\end{array}$
\end{tabular} \\
\hline
\end{tabular}

fécondité). Les biomarqueurs biochimiques les plus couramment utilisés sont [2]: les métallothionéines dont la teneur dans les cellules est liée à la présence de certains métaux dans le milieu, les protéines de stress, les marqueurs de stress oxydatif (atteintes radicalaires de certaines macromolécules ou de certaines fonctions cellulaires), l'activité acétylcholinestérase $(\mathrm{AChE})$ inhibée par la présence de certains contaminants (métaux lourds et pesticides), les monooxygénases à cytochromes P450 liées à la présence de HAP, dioxines, PCB, les enzymes de conjugaison (ex: Glutathione S-transferase: GST) impliquées dans l'excrétion de métabolites toxiques.

Les mesures de ces biomarqueurs peuvent être réalisées sur des organismes test de manière standardisée pour permettre une stabilité satisfaisante des mesures mais cela soulève la question de la représentativité vis-à-vis des systèmes écologiques naturels (Blandin, 1986). En effet, les paramètres du milieu et leur variabilité d'une part, et la diversité génétique des espèces, d'autre part, ne sont pas pris en compte par ce type d'expérimentation. L'autre approche est celle de la mesure in situ où les organismes intègrent les modifications du milieu.

C'est dans ce cadre que nous développerons plus en détail le biomarqueur EROD et son utilisation concrète in situ.

\section{LE BIOMARQUEUR EROD}

Suite à une contamination du milieu aquatique, les polluants peuvent pénétrer à l'intérieur des organismes par voie directe (exposition) et/ou par voie alimentaire, se propager, s'accumuler au niveau de différents tissus et exercer leur toxicité sur l'organisme cible. Cependant, un système de détoxication des xénobiotiques s'est mis en place au cours de l'évolution: il transforme les xénobiotiques bioaccumulables, en général hydrophobes, en produits hydrophiles qui peuvent être éliminés plus facilement dans l'urine et la bile.

Dans une première phase de la biotransformation interviennent des complexes multienzymatiques: les monooxygénases à cytochrome P450. Une deuxième phase augmente encore la solubilité des produits qui sont alors rapidement éliminés par l'organisme.

Chez le poisson, les activités de biotransformation de phase I peuvent être quantifiées en particulier par la mesure de l'activité Ethoxy-Resorufine O-deethylasique
(EROD) dans le foie, mais de nombreuses autres mesures d'activités enzymatiques sont citées dans la littérature scientifique ([4]...).

Cette activité EROD est augmentée (on parle d'induction) par l'exposition à un certain nombre de molécules chimiques. Cette classe d'inducteurs regroupe des molécules généralement très toxiques mais aussi stables et lipophiles, qui s'accumulent facilement dans les organismes :

- des composés hydrocarbures aromatiques polycycliques ou HAPs comme le 3-méthylcholanthrène, le benzo(a)pyrène, le benzo(a)anthracène ;

- des hydrocarbures aromatiques halogénés très toxiques tels que la 2,3,7,8-tétrachlorodibenzo(p)dioxine ou TCDD. On les trouve à l'état de traces dans les préparations commerciales de substances chlorées (pesticides...) et dans certains effluents de papeteries [5];

- des polychlorobiphényls ou PCBs plans [6]; tels que les isomères 3, 3'4,4'-tétrachlorobiphényl ou PCB 77 et 2,3' 4,4'5-pentachlorobiphényl ou PCB 118 ;

- des inductions ont été observées pour des effluents complexes (industriels et municipaux)...

Outre la spécificité à une catégorie de xénobiotiques, l'induction présente les trois caractéristiques suivantes : rapidité (délai de 2 à 5 jours après l'exposition), concentrationdépendance, réversibilité.

Les activités monooxygénases peuvent à la fois réduire la toxicité des polluants (c'est leur rôle principal) mais aussi augmenter leurs effets chroniques ou cancérigènes lorsque c'est le composé biotransformé qui présente ces dangers pour l'organisme. Dans ce cas une induction des activités monooxygénases est prédictive d'un risque réel pour les organismes. Dans tous les autres cas elle indique uniquement l'exposition des organismes à des polluants.

Chez les poissons exposés aux polluants chimiques, le système de biotransformation est sollicité et les activités enzymatiques de détoxication sont induites. Mais indépendamment de toute pollution, les activités biochimiques, dont l'EROD, sont soumises à l'influence de nombreux autres facteurs dont il faut connaître avec précision les influences afin d'interpréter correctement les niveaux du biomarqueur EROD mesuré sur des poissons prélevés dans le milieu.

On observe généralement des niveaux d'activités monooxygénases différents selon les espèces, le sexe et la maturité sexuelle des poissons (les mâles présentent des activités légèrement supérieures aux femelles ([8] chez $O$. mykiss ; [9] chez Chondrostoma nasus). La température du 
Tableau 2. - Principaux facteurs modulateurs naturels (d'après [7]).

\begin{tabular}{|c|c|}
\hline Espèce & Age \\
Lignée & Etat reproductif \\
Sexe & Température \\
\hline
\end{tabular}

milieu est également un facteur important dans la mesure où elle gouverne la température interne des poissons et leur maturation sexuelle au long de l'année. A ces facteurs environnementaux naturels vient s'ajouter la pollution chimique.

Afin d'atténuer les effets des facteurs de modulation sur le diagnostic, il est possible de calculer un taux d'induction qui est défini comme le rapport de l'activité EROD de l'échantillon considéré sur l'activité moyenne, dite de référence, mesurée sur les poissons de même espèce, de même sexe et pêchés à la même saison sur la station de référence.

L'utilisation d'activités monooxygénases pour identifier les zones de pollutions chimiques dans l'environnement aquatique s'est développée au cours des vingt dernières années dès 1976 [4]. Des taux élevés dans les foies de poisson ont été observés au niveau des zones affectées par des déversements de pétrole [10], [4] ou par des effluents d'usines (ex. : papeteries, usines d'incinération de PCBs) [11], [12].

Un certain nombre d'études ont concerné les zones côtières américaines [13] ou françaises [14], [15], [16]. En France, après plusieurs années d'expérimentation, l'activité EROD est actuellement envisagée comme outil de biosurveillance des côtes françaises au sein du Réseau National d'Observation de la qualité du milieu marin, le RNO [17].

\section{EXEMPLES D'UTILISATION DE L'EROD POUR LA SURVEILLANCE DES EAUX DOUCES}

\subsection{La Durance}

Le principal pôle industriel de la moyenne Durance est une usine chimique installée à Saint-Auban. Cette usine est une importante unité de production qui produit essentiellement du chlore et du chlorure de vinyle mais aussi du trichloréthylène, du trichloréthane, de l'acide monochloracétique et du polychlorure de vinyle.

Les résultats des nombreuses analyses effectuées en 1991 et 1992 au niveau de l'eau et des matières en suspension mettent en évidence la présence de certains éléments toxiques comme les métaux, les solvants chlorés produits par l'usine de Saint-Auban mais aussi des pesticides précis: l'hexachlorobenzène, les congénères $\alpha, \beta$ et $\gamma$ d'hexachlorocyclohexane $(\mathrm{HCH})$.

La mesure de l'activité EROD a été utilisée en 1991 afin de caractériser la qualité des milieux aquatiques le long de la Durance [3] et compléter une étude similaire réalisée

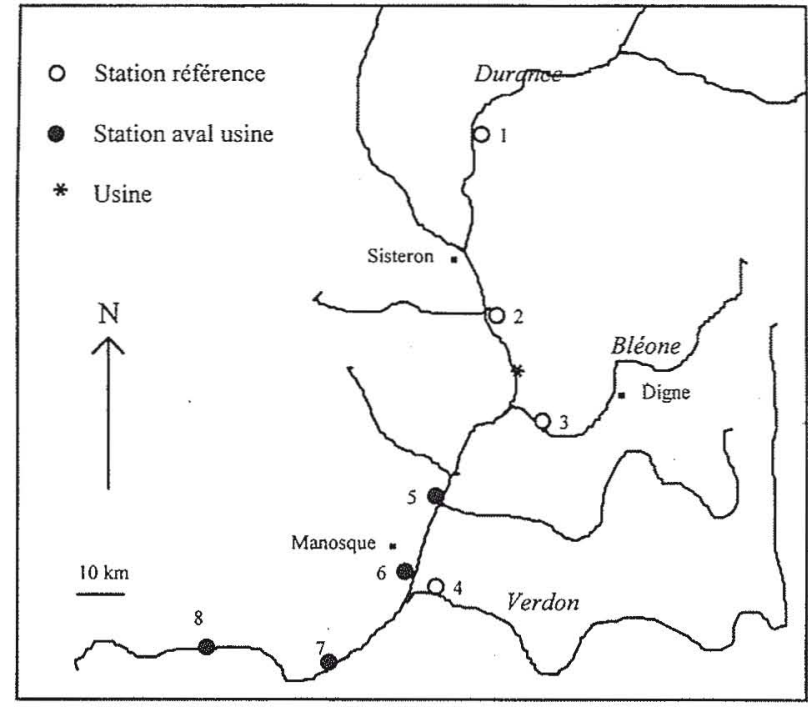

1. Carte de la Durance et des stations de pêche.

auparavant [18]. Huit stations ont été choisies pour cette étude :

- quatre stations de "référence»: sur la Durance, en amont de l'usine de Saint-Auban (stations 1 et 2), et sur deux affluents de la Durance, la Bléone et le Verdon (stations 3 et 4 ) ;

- quatre stations sur la Durance en aval de l'usine: Brillanne, Manosque, Peyrolles et Roque d'Anthéron (respectivement stations $5,6,7$ et 8 ).

Trois espèces de Cyprinidés ont été retenues en raison de leur abondance dans la Durance, la Bléone et le Verdon, et ont été capturées lors de pêches à l'électricité : Barbus barbus (le barbeau commun), Leuciscus cephalus (le chevaine) et Chondrostoma nasus (le hotu).

L'activité EROD, pour chaque individu, est ensuite exprimée en taux d'induction EROD calculé par rapport à l'activité de référence (fig. 2).

L'analyse statistique globale met en évidence un effet station significatif (Kruskal et Wallis, $p=0,000$ et l'analyse statistique détaillée montre que chaque station à l'aval de l'usine se distingue des autres. Globalement, un gradient décroissant d'induction est visible au fur et à mesure que l'on s'éloigne de l'usine. L'induction est maximale pour les trois espèces à la station 5 , la plus proche de l'usine. Ensuite les profils d'induction par espèce se différencient.

Le barbeau est induit de façon significative même à la station 8, la plus éloignée de l'usine contrairement aux deux autres espèces. La même remarque peut être faite au sujet du hotu pour la station 7. En revanche les hotus sont les seuls poissons non induits à la station 6 . L'espèce chevaine ne présente plus d'induction dès la station 7 .

Les PCBs, potentiels responsables de l'induction, ont été recherchés dans le foie des poissons au printemps 1993. Il est apparu qu'aucun des 20 poissons pêchés aux stations 2 et 3 n'a accumulé dans son foie de PCBs en quantité détectable. Ce n'est pas le cas des poissons pêchés en aval de l'usine (stations 5 et 6) dont seulement une minorité ne présentent pas de PCBs. Par ailleurs, la Brillanne (station 5) affiche une teneur en PCB moyenne 


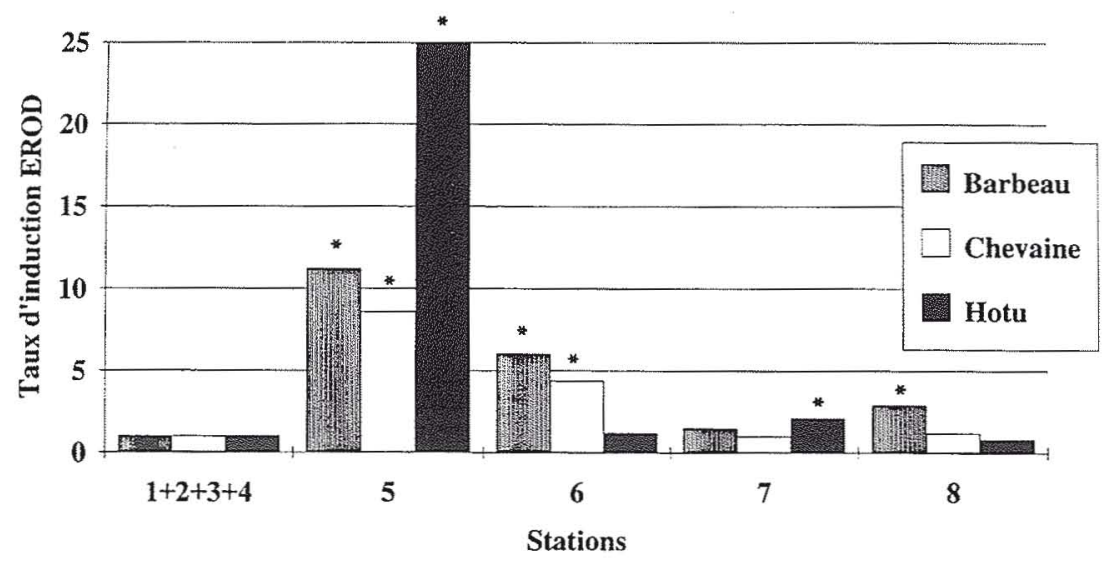

2. Taux d'induction EROD moyen annuel par espèce et par station sur la Durance en 1991 [3].

Les étoiles indiquent des différences significatives par rapport aux stations de référence $(p<05)$.

plus élevée que Manosque (station 6) qui suit donc l'évolution de l'induction (respectivement 1056 et $586 \mu \mathrm{g}$ $\mathrm{PCB} / \mathrm{kg}$ de poids $\mathrm{sec}$ ).

Il est intéressant de constater que les barbeaux présentent dans leur foie, des teneurs en PCBs bien supérieures à celles dosées dans les chevaines. Leur comportement benthique explique certainement leur forte contamination, les sédiments étant en principe fixateurs de micropolluants.

L'étude de 1991 a ainsi permis de mettre en évidence un impact des rejets d'une usine chimique de la Durance sur l'activité biochimique EROD dans le foie des poissons pêchés à $20 \mathrm{~km}$ et plus. De fortes inductions sont constatées chez les trois espèces de Cyprinidés pêchées. Le taux d'induction se révèle lié à la teneur en PCBs totaux dosée dans le foie de chaque poisson.

\subsection{La Moselle}

Une étude réalisée en 1992-1993 sur la Moselle, avec le soutien de l'Agence de l'eau Rhin-Meuse, a eu pour objectifs d'évaluer l'écotoxicité des effluents les plus importants du secteur (amont et aval d'Epinal) et leur impact sur les populations piscicoles en place [19].
Des prélèvements de sédiments ont été réalisés en mai 1995 sur 5 des stations étudiées. Ils révèlent la présence de HAPs sur toutes les stations prospectées. En ce qui concerne

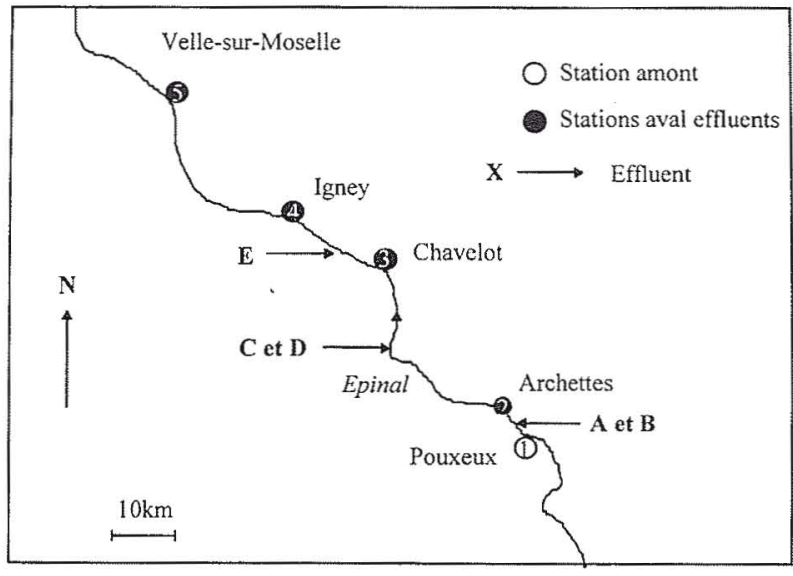

3. Carte de la Moselle, des stations de pêche et de rejets industriels majeurs.

Tableau 3. - Présentation des principaux effluents rejetés sur le secteur étudié de la Moselle.

\begin{tabular}{|c|c|c|}
\hline Code & Type & Caractéristiques \\
\hline rejet $\mathrm{A}$ & Papeterie & $\begin{array}{l}\text { Pas de blanchiment au chlore } \\
\text { Pas de traitement des eaux résiduaires }\end{array}$ \\
\hline rejet $B$ & Papeterie & $\begin{array}{l}\text { Pas de blanchiment au chlore } \\
\text { Utilisation de colorants } \\
\text { Epuration primaire }\end{array}$ \\
\hline rejet $\mathrm{C}$ & Station d'épuration & Limite de norme \\
\hline rejet $\mathrm{D}$ & Usine de production de pâte à papier & $\begin{array}{l}\text { Pas de blanchiment au chlore } \\
\text { Epuration primaire et secondaire }\end{array}$ \\
\hline rejet $E$ & Teinturerie & $\begin{array}{l}\text { Utilisation de colorants } \\
\text { Décantation }\end{array}$ \\
\hline
\end{tabular}


les métaux, le zinc, le plomb, le chrome, le mercure et le cuivre sont détectés à des concentrations supérieures aux concentrations de référence. Parmi les 17 pesticides organochlorés recherchés, six seulement ont été détectés. En ce qui concerne les $\mathrm{PCBs}$, quatre congénères sur les sept recherchés sont détectés, dont deux présents à toutes les stations (PCBs 138 et 153 ).

Les trois mêmes espèces de Cyprinidés ont été prélevées en raison de leur abondance sur l'ensemble des stations étudiées: le barbeau, le chevaine et le hotu.

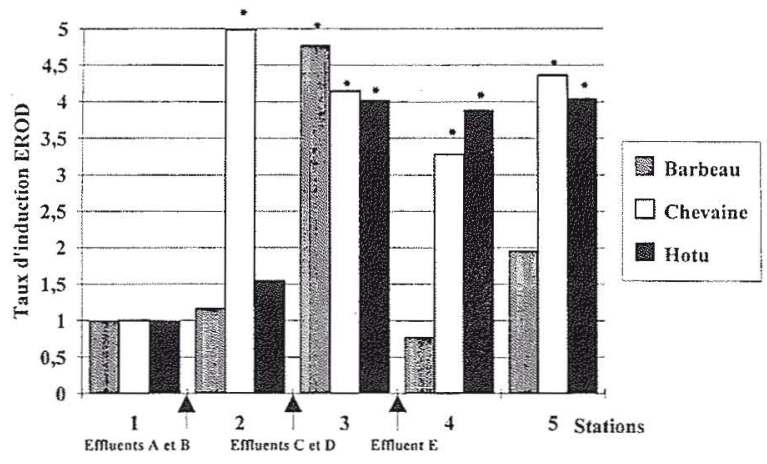

4. Taux d'induction EROD moyen annuel par espèce et par station sur la Moselle en 1992 [3].

Les étoiles indiquent des différences significatives par rapport à la station $1(p<05$

Si on compare statistiquement les taux d'induction moyens calculés par station, toutes espèces confondues, un effet station est mis en évidence $(p<0,01)$ et l'analyse statistique de comparaison de moyennes deux à deux (Mann et Whitney) permet de discriminer deux groupes de stations : la station située en amont de tous les rejets étudiés, Pouxeux (station 1), est statistiquement différente des trois stations situées en aval d'Epinal, Chavelot, Igney et Velle-surMoselle (respectivement 3, 4 et 5).

Les taux d'induction moyens, calculés à chaque station et pour chaque espèce, sont présentés sous formes d'histogrammes dans la figure 4 qui met en évidence des variations interspécifiques du taux d'induction en fonction des stations.

L'espèce chevaine présente des inductions significatives dès la station 2 et jusqu'à la dernière station. Les hotus et les barbeaux, contrairement aux chevaines, ne présentent pas d'induction d'activité EROD en aval des rejets $A$ et $B$, station 2. L'activité des hotus est induite sur les stations 3, 4 et 5 alors que les barbeaux n'affichent une induction significative qu'à la station 3 .

Quelle que soit l'espèce considérée, à l'aval d'Epinal (station 3) on peut considérer une induction particulièrement marquée. Les poissons sont induits jusqu'à la station le plus à l'aval à une trentaine de kilomètres du dernier rejet $E$ alors que nos informations ne font pas état d'autre rejet important à ce niveau.

Des études parallèles ont été réalisées en laboratoire sur des truites immatures exposées durant 96 heures à des gradients de concentrations pour chacun des 5 effluents. Les effets mesurés en laboratoire s'accordent avec les réponses d'induction EROD obtenues in situ en aval des rejets étu- diés. Cependant, hormis pour le rejet E, nous n'observons pas de relation dose-effet simple.

Le rejet E (teinturerie) se distingue des autres par un pouvoir inducteur plus élevé puisque l'induction est signifícative dès la plus faible concentration d'essai $(0,5 \%)$. L'effet inducteur de cet effluent pourrait être lié en partie, à la présence de molécules colorantes de structure proche de substances telles que le 3,3',4,4-tétrachloro-azobenzène, décrites comme inductrices des P450IA chez le rat [20].

En définitive, sur le secteur étudié, la Moselle reçoit 5 effluents principaux dont les potentiels toxiques et les capacités à induire l'activité EROD des poissons ont été mis en évidence au cours d'expériences en laboratoire. In situ, le biomarqueur EROD se montre suffisamment sensible pour signaler par une induction, cohérente avec l'étude de laboratoire, une contamination des poissons accrue en aval des premiers rejets étudiés et jusqu'à la dernière station.

\section{$\mathrm{V}$ 밈 CONCLUSION}

Sur la Durance, l'augmentation de l'activité EROD a traduit une influence des rejets chimiques de l'usine sur les poissons pêchés jusqu'à $30 \mathrm{~km}$ en aval. Sur la Moselle, l'utilisation de l'activité EROD in situ, comme en laboratoire, a permis de mettre en évidence l'impact de plusieurs effluents industriels et urbains sur la qualité de l'eau.

Nos résultats confirment ainsi la sensibilité du biomarqueur EROD puisque sa réponse est modulée de façon significative en fonction du niveau de contamination.

Parallèlement aux mesures d'induction EROD, étaient effectués des examens des peuplements d'invertébrés au cours des deux études. On a constaté que le diagnostic donné par le biomarqueur EROD était cohérent avec celui donné par l'étude des invertébrés [3].

Pour l'utilisation in situ du biomarqueur EROD, il est très important de bien choisir la ou les stations de référence car ce choix détermine la mise en évidence d'un impact. Si l'étude consiste à mettre en évidence l'impact spécifique d'un rejet précis (ce qui est généralement le cas), la meilleure situation est alors en amont de ce rejet, en veillant à ce qu'aucune autre source polluante n'intervienne entre cette station de référence et la station située en aval du rejet (en pratique, ceci est difficile à obtenir).

Par ailleurs, afin de supprimer les difficultés d'échantillonnage sur certains sites, plusieurs études ont utilisé des poissons d'élevage placés dans des cages et disposés au niveau de stations prédéterminées. Cette technique résout le problème de la mobilité des poissons sur le site. Le marqueur biochimique procure ainsi une information plus précise du niveau de contamination à chaque station, ce qui favorise l'identification des sources de polluants. Elle permet d'utiliser une espèce sentinelle qui peut donner une information objective sur la qualité de l'eau, en ce sens que les poissons introduits dans le milieu ne présentent pas les phénomènes d'adaptation à la pollution ambiante que peuvent présenter les espèces locales [5].

Enfin, le dosage de l'activité EROD nécessite des précautions lors du prélèvement des foies et de sa conservation sur le terrain [7]. C'est pourquoi, la technique d'immunodétection de la teneur en P450IAl est certainement une méthode qui permettra un échantillonnage plus facile et une détection automatisée [21], [7], [2]. Cette méthode immunochimique 
confirme mais également complète l'information apportée de façon précoce par les activités catalytiques sur le niveau d'induction du P450IA dans le poisson. En particulier, l'immunodétection permet de détecter les enzymes P450IA dont l'activité catalytique est inhibée par un polluant ou qui ont perdu leur capacité catalytique. Elle se révèle donc d'un intérêt considérable pour l'interprétation et la compréhension des comportements de faible ou «non induction » d'activité EROD.

Actuellement, les gestionnaires soucieux de l'environnement disposent d'une palette d'outils. Les méthodes de bioévaluation de la qualité de l'eau sont, en effet, variées et interviennent à différents niveaux d'organisation biologique. Elles apportent des informations différentes et souvent complémentaires. Etant donné l'intérêt de la mesure de l'activité EROD et pour favoriser son développement, il paraît indispensable d'engager dès à présent, des études de standardisation et d'intercalibration de la méthode [7]. Cette mesure est actuellement proposée à titre expérimental pour 4 ans dans le Réseau National d'Observation de la qualité des côtes françaises. En eau douce, les Finlandais et les Suédois valident actuellement l'utilisation de l'activité EROD pour la surveillance des effluents de papeteries.

\section{Bibliographie}

[1] Huggett R.J., Kimerle R.A., Mehrle P.M. \& Bergman H.L. (1989). — «iomarkers : biochemical, physiological and histological markers of anthropogenic stress », SETAC Special Publications Series, Lewis Publishers, Chelsea, US, 347 p.

[2] Vindimian E. \& Garric J. (1992). - Bioessais et bioindicateurs de toxicité dans le milieu naturel. Ministère de l'Environnement. Etude inter agences n ${ }^{\circ}$ 17-61 p.

[3] RiCHERT C. (1994). - Comparaison de marqueurs biochimiques chez les poissons et d'indicateurs écologiques pour le diagnostic in situ de la pollution toxique dans les cours d'eau. Thèse de I'Université Claude Bernard-Lyon I, 190 pp.

[4] PAYNe J.F. (1976). - Field evaluation of benzopyrene hydroxylase induction as a monitor for marine petroleum pollution. Science, 191, 945-946.

[5] LINDSTRÖM-SEPPÄ P. \& OIKARI A. (1990). - Biotransformation and other toxicological and physiological responses in rainbow trout (Salmo gairdneri Richardson) caged in a lake receiving effluents of pulp and paper industry. Aquatic Toxicology, 16, 187-204.

[6] Melancon M.J., Elcombe C.R., Vodicnik M.J. \& LeCh J.J. (1981). - Induction of cytochromes P450 and mixed function oxidase activity by polychlorinated biphenyls and $\beta$-naphtoflavone in carp, Cyprinus carpio. Comp. Biochem. Physiol., 69C, 219-226.

[7] Goksøyr A. \& FörLin L. (1992). - The cytochrome P-450 system in fish, aquatic toxicology and environmental monitoring. Aquatic Toxicology, 22, 287-312.

[8] FörLin L. \& HauX C. (1990). - Sex differences in hepatic cytochrome $\mathrm{P}-450$ monooxygenase activities in rainbow trout during an annual reproductive cycle. Journal of Endocrinology, $124,207-213$.

[9] Masfaraud J.F., Monod G. \& Devaux A. (1990). - Use of the fish cytochrome P-450-dependent 7-ethoxyresorufin O-deethylase activity as a biochemical indicator of water pollution. Study of the liver and the kidney of male and female nase (Chondrostoma nasus) from the river Rhône. The Science of the Total Environment, 97/98, 729-738.
[10] Payne J.F. \& Penrose W.R. (1975). - Induction of aryl hydrocarbon (benzo(a)pyrene) hydroxylase in fish by petroleum. Bull. Environ. Contam. Toxicol., 14/1, 112-116.

[11] Monod G., Devaux A. \& Riviere J.L. (1988). - Effects of chemical pollution on the activities of hepatic xenobiotic metabolizing enzymes in fish from the river Rhone. Comp. Biochem. Physiol., 88C/1, 83-89.

[12] LindströM-SEPpä P. \& Oikari A. (1988). - Hepatic xenobiotic biotransformation in fishes exposed to pulpmill effluents. Wat. Sci. Tech. 20/2, 167-170.

[13] Stegeman J.J., Woodin B.R. \& Goksøyr A. (1988). - Apparent cytochrome $\mathrm{P}-450$ induction as an indication of exposure to chemicals in the flounder Platichthys flesus. Mar. Ecol. Prog. Ser., 46, 55-60.

[14] Lafaurie M., Giudicelli J., Carriere S., Lemaire P., Mathieu A. \& Negre Y. (1989). - Pollutant biotransformation in marine teleost fish: Use in environmental health evaluation. Advances In Applied Biotechnology Series, 5, 141-152.

[15] Galgani F., Boceuene G., Lucon M., Grzebyk D., Letroutt F. \& ClaISSE D. (1991). - EROD measurements in fish from the northwest part of France. Marine Pollution Bulletin, 22/10, 494-500.

[16] Narbonne J.F., Garrigues P., Ribera D., Raoux C., Mathieu A., Lemaire P., Salaun J.P. \& Lafaurie M. (1991). — Mixedfunction oxygenase enzymes as tools for pollution monitoring: field studies on the french coast of the Mediterranean sea. Comp. Biochem. Physiol., 100C/1-2, 37-42.

[17] Cl.aisse D., Joanny M. \& Quintin J.Y. (1992). - Le réseau national d'observation de la qualité du milieu marin (RNO). Analysis Magazine, 20/6, 19-23.

[18] Vindimian E., Namour P., Migeon B. \& Garric J. (1991). In situ pollution induced cytochrome P-450 activity of freshwater fish : barbel (Barbus barbus), chub (Leuciscus cephalus) and nase (Chondrostoma nasus). Aquatic Toxicology, 21, 255266.

[19] Garric J., Bray M., Migeon B., Vollat B. \& Richert C. (1994). - Evaluation de l'écotoxicité de rejets industriels et urbains et de la contamination du milieu récepteur, rapport Cemagref, 33 p.

[20] Bunce N.J., Choong D.K.M., Landers J.P. \& ZaCharewski T.R. (1989). - Competitive binding of chlorinated azobenzenes to the Ah receptor protein. Environ. Toxicol. Chem., 8, 25-30.

[21] GoKsøYR A. (1991). - A semi-quantitative cytochrome P450IA1 ELISA : a simple method for studying the monooxygenase induction response in environmental monitoring and ecotoxicological testing of fish. The Science of the TOTAL ENVIRONMENT, 101, 255-262.

[22] LINDSTRÖM-SEPPÄ P. \& OIKARI A. (1989). - Biotransformation and other physiological responses in whitefish caged in a lake receiving pulp and paper mill effluents. Ecotoxicology and Environmental Safety; 18, 191-203.

[23] LINDSTRÖM-SEPPÄ (1990). - Biotransformation in fish : Monitoring inland water pollution caused by pulp and paper mill effluents. Publications of the university of Kuopio, Natural Sciences, original Reports, 69 pp.

[24] Stegeman J.J. (1981). - Polynuclear aromatic hydrocarbons and their metabolism in the marine environment. Polycyclic hydrocarbons and cancer (GELBOIN H.V. ET TSO, P.D.P., EDS.), ACADEMIC PRESS, NEW-YORK, 3, 1-60.

[25] Stegeman J.J. (1989). - Cytochrome P-450 forms in fish: catalytic, immunological and sequences similarities. Xenobiotic $a, 19 / 10,237-245$ 